\title{
METHODS OF DETERMINATION OF THE SIGN OF THE THERMO-DIFFUSION COEFFICIENT IN DISPERSED LIQUIDS
}

In this paper three methods of determination of the sign of the thermo-diffusion coefficient (Soret constant) are presented. The first method uses the relation for Soret constant which was derived from the thermo-diffusion equation. This relation permits determination of the sign of the Soret constant as well as its approximate value. The second method is based on the time dependence of the decay of the created diffraction grating. The last method is based on the measurement of the transmission of light by the dispersion liquid.

\section{Introduction}

When the nanoparticles with the diameter of $(10 \div 20) \mathrm{nm}$ are placed in a liquid medium they are dispersed and at the same time a temperature and concentration gradient are created. As a result there will be forces acting on the particles and, therefore, the diffusion and thermo-diffusion flux of the particles will rise. According to the first Fick law the value of the density of flux of the nanoparticles in one-dimensional case can be expressed by the equation

$$
i=-D \cdot \frac{\partial n}{\partial x}-D \cdot S \cdot n \cdot \frac{\partial T}{\partial x}
$$

where the first expression on the right hand side is the diffusion flux and the second one on the right hand side is the thermodiffusion flux of the particles. $D$ is the diffusion constant of the particles, $S$ is the thermo-diffusion coefficient (the Soret constant) and $T$ is temperature. It is possible to show experimentally that if a gradient of temperature is created in the sample, the particles can accumulate, (when specific conditions are fulfilled), at the place where the temperature is lower $(S>0)$ or at the place where the temperature is higher $(S<0)$.

The explanation of the reason why $S<0$ is known for gasses, but for liquids a satisfying theory is unknown. That is the reason why there is permanent interest in the solution of this problem in the world [1], [2].

In this paper we present three methods used for the determination of the sign or value of the thermo-diffusion coefficient.

\section{Arrangement of an experimental apparatus}

For the determination of the sign and value of the Soret constant the experimental apparatus which is depicted in Fig. 1 was used.

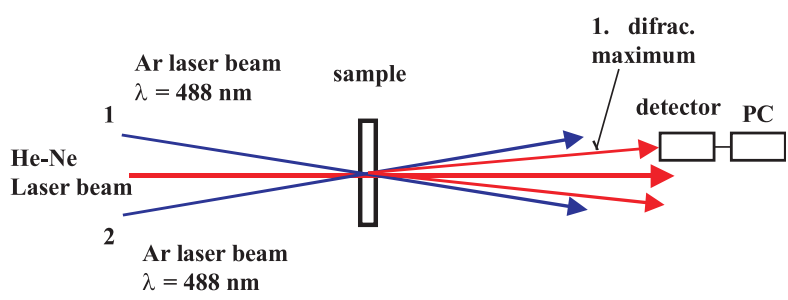

Fig. 1 Schematic arrangement of the experimental apparatus.

In order to create the particle diffraction grating and also to increase the temperature of the disperse liquid at the place of measurement the Ar laser beam with $\lambda=488 \mathrm{~nm}$ and $(0 \div 120) \mathrm{mW}$ power was used. To create the diffraction grating, the laser beam was divided into two crossed beams. At the point where the two beams are crossing the periodical light field arises due to their interference. The periodical thermal field arises because the light energy is absorbed in the sample. Consequently, due to the diffusion and thermo-diffusion of the nanoparticles the particle diffraction grating in the liquid is created.

\section{Methods of measurements}

a) Method of direct calculation

If we take the sample in the form of a thin layer of the investigated liquid and create the temperature difference $\Delta T=T_{2}-T_{1}$ between two places with coordinates $x_{1}$ and $x_{2}$, the stationary concentration difference $\Delta n$ arises. Eq. (1) for the stationary case gives

$$
i=0=-D \cdot \frac{\partial n}{\partial x}-S \cdot D \cdot n \cdot \frac{\partial T}{\partial x} .
$$

After solving this equation for $S$ we obtain

$$
S=\frac{1}{n} \cdot \frac{d n}{d T} \text {. }
$$

\footnotetext{
* Ctibor Musil, Július Štelina

Department of Physics, Faculty of Electrical Engineering, Žilina University , Univerzitná 1, 01026 Žilina, Slovak Republic E-mail: stelin@fel.utc.sk
} 
If we assume a small linear change of temperature which is connected with small changes of concentration then the relation (3) can be expressed as

$$
S=\frac{1}{n} \cdot \frac{\Delta n}{\Delta T} .
$$

The experimental determination of the local value of $n$ is not simple. This one is, however, closely related with the light absorption coefficient $\alpha$ which can be measured directly. Using the equation

$$
\frac{\Delta n}{n}=\frac{\Delta \alpha}{\alpha}
$$

one gets for the Sorret constant

$$
S=\frac{1}{\alpha} \cdot \frac{\Delta \alpha}{\Delta T}
$$

where all the quantities necessary for the evaluation of $S$ may be obtained from the experiment.

Moreover, the absorption coefficient can be determined from the relation

$$
\alpha=\frac{1}{L} \cdot \ln \left|\frac{U}{U_{1}}\right|,
$$

where $L$ is the sample thickness, $U$ is the signal produced by the detector when the laser beam is passing across the empty cell and $U_{1}$ is the signal produced by the detector when the laser beam is passing through the cell in which the sample is placed. After substituting $U$ expressed by equation (7) into relation (6), we obtain

$$
S=\frac{1}{\Delta T} \cdot \frac{\ln \left(\frac{U_{1}}{U_{2}}\right)}{\alpha \cdot L},
$$

where $U_{1}, U_{2}$ are signals from the detector after the laser beam passed through the sample at the temperatures $T_{1}, T_{2}$, respectively [3].

b) The time dependence method of the decay of the grating

If we take the sample in the form of a thin layer of colloidal liquid (thickness of $60 \mu \mathrm{m}$ ) in the interference field of two laser beams (Fig. 1), due to the absorption of light the temperature grating in the sample will be created and the nanoparticles will start repartitioning in the temperature field created according to the sign of the thermo-diffusion coefficient (Soret constant $S$ ) and they will start to create an absorption grating [4].

After cutting out the interference field of the crossed beams, the temperature grating will vanish in about $3 \mathrm{~ms}$ and the diffraction absorption grating will decay as depicted in Fig. 3.

If one screens one beam of the Ar -laser only the remaining laser beam will be absorbed in places of particle accumulation (grating) and the temperature in these places will remain higher than in the places where the particles concentration is lower. If $S<0$, the thermo-diffusion flux will be greater than the diffusion flux and the grating decay will be slowed down or the grating will even be reconstructed (Fig. 2) [4]. From the behaviour of the time dependence depicted in Fig. 3 it follows that $S>0$ and the effective diameter of the nanoparticles can also be determined.

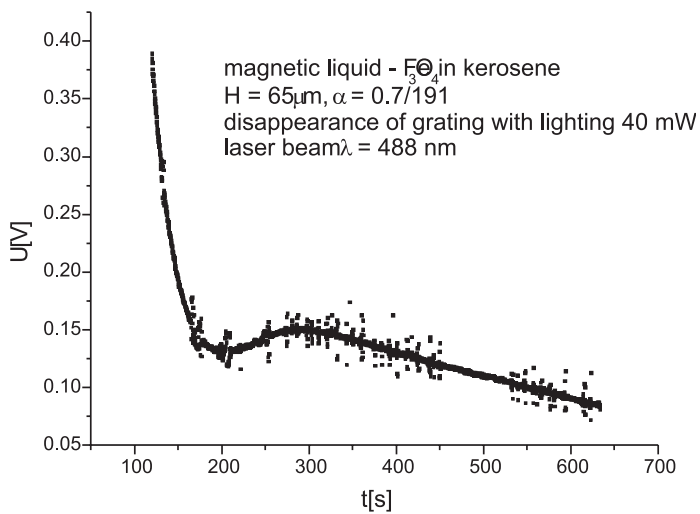

Fig. 2 The time dependence of decay of the lighting particles grating when $S<0$.

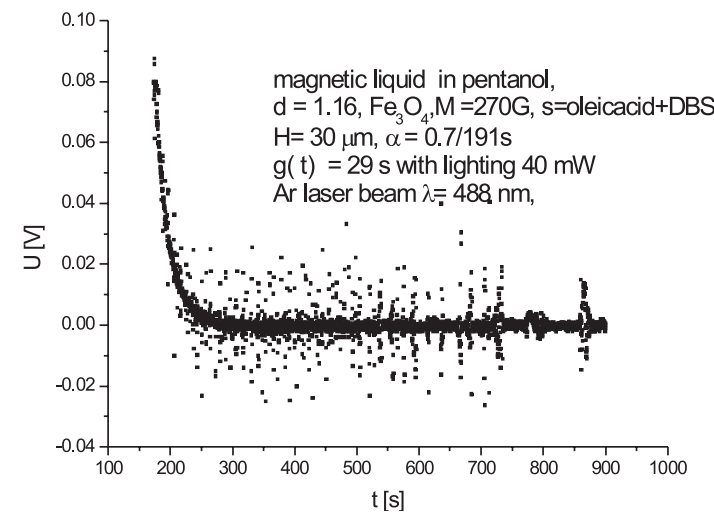

Fig. 3 The time dependence of decay of the lighting particles grating when $S>0$.

c) Method of measurement of light transmission

In this method the sample of disperse liquid of the thickness cca $(60 \div 100) \mu \mathrm{m}$ is being heated by means of the absorption of the laser beam of high intensity at the point of its passing through the sample (Fig. 4). In this same place the diagnostic beam (e. g. $5 \mathrm{~mW} \mathrm{He}-\mathrm{Ne}$ laser beam) passes through the sample as well.

In the case $S>0$ the particles are ejected from the place of heating [4] and the intensity of the diagnostic beam grows. If $S<0$, the dispersed particles accumulate in the place of heating and the intensity of the diagnostic beam decreases (Fig. 5).

\section{Experimental results}

For the disperse liquid with nanoparticles $\mathrm{Fe}_{3} \mathrm{O}_{4}$ based on kerosene at the temperature $24{ }^{\circ} \mathrm{C}$ we found $S<0$ with value 


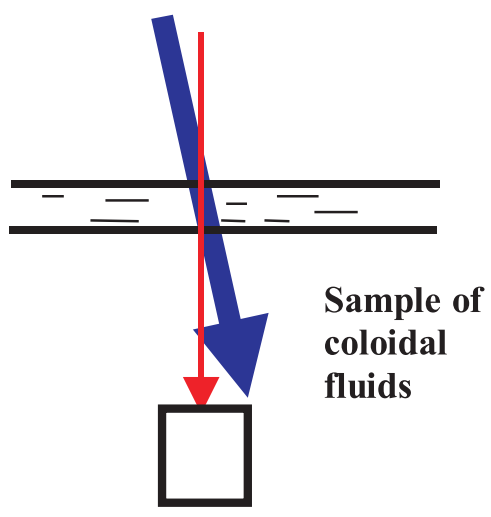

Fig. 4 Schematic arrangement for reading dates to determine the light absorption in the sample.

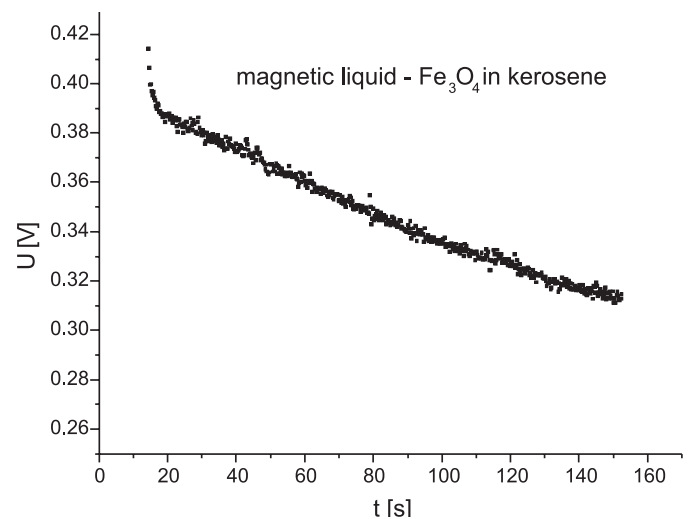

Fig. 5 The time dependence of detection signal through the sample $(S<0)$ if that is heating.

approximate magnitude of the Soret constant. The accuracy of the first method depends mainly on the accuracy with which the initial and final temperatures of the liquid $T_{1}$ and $T_{2}$ and the voltages $U_{1}$ and $U_{2}$ are determined in the stationary regime of the liquid sample in the presence of the Ar laser beam.

Except for the fast determination of the sign of the Soret constant, these methods can also serve for the study of the thermo-diffusion and kinetic phenomena connected with it in the liquids in which nanoparticles are present.

This work has been supported by project VEGA.

\section{References}

[1] LANDAU, L. D., LIFŠIC, E. M.: Hydrodynamics (in Russian), Moskva, Nauka 1986

[2] DE GANS, B., KITA, R., WIEGAND, S., LUETTMER-STRATHMANN, J.: Phys. Rev., Lett. 91, 2455501, 2003

[3] ŠTELINA, L., MUSIL, C.: Metod for specification sign and value of Soret constant factor in colloid fluids (in Slovak), ADVANCES in Electrical and Electronic Engineering, ŽU, No.1, Vol. 3/2004, Žilina, pp. 55-58

[4] ŠTELINA, J., MUSIL, C., BRACINÍK, J., KOPČANSKÝ, P., TIMKO, M., KONERACKÁ, M.: Influence of the sign of Soret constant on the formation and decay of the nanoparticle structures in dispersion fluids, $11^{\text {th }}$ International Workshop on APCOM, Malá Lučivná, 2005, pp. 230-234

[5] KOPČANSKÝ, P., TIMKO, M., POTOČOVÁ, I., KONERACKÁ, M., JURÍKOVÁ, A., TOMAŠOVIČOVÁ, N., ŠTELINA, J., MUSIL, C., BRACINÍK, J.: The determination of the hydrodynamic diameter of magnetic particles using FRS experiment, Jour. of Magnetism and Magnetic Materials, 289, pp. 97-100, 2005 